\title{
Geriatric nutritional risk index predicts surgical site infection after pancreaticoduodenectomy
}

\author{
NAOTAKE FUNAMIZU, YUKIO NAKABAYASHI, TOMONORI IIDA and KAZUNAO KURIHARA \\ Department of Digestive Surgery, Kawaguchi Municipal Medical Center, Saitama, Saitama 333-0833, Japan
}

Received April 19, 2018; Accepted July 10, 2018

DOI: $10.3892 / \mathrm{mco} .2018 .1671$

\begin{abstract}
Surgical site infections (SSIs) are a well-known potential complication of surgery. They are assocaited with preoperative malnutrition and lead to increased medical costs and longer hospital stays. Therefore, surgeons should appropriately identify patients who are at a high risk. The geriatric nutritional risk index (GNRI) is a tool, increasingly utilized to assess the degree of malnutrition, particularly in elderly patients. Therefore, the present study attempted to validate whether GNRI could predict the risk of SSI in patients following pancreaticoduodenectomy (PD). A cohort study was retrospectively conducted on 106 patients in the Department of Digestive Surgery, Kawaguchi Municipal Medical Center, Japan from January 2007 to December 2017. All patients were subjected to nutritional screening using GNRI and followed up for the occurrence of postoperative complications, including SSI post PD. Additionally, risk factors for developing SSI, and the patient's height, body mass index and preoperative laboratory values were documented. Patients were divided into SSI $(n=15)$ and non-SSI $(n=91)$ groups with a determined incidence of $14.2 \%(15 / 106)$ for SSI. The results revealed that the SSI group had GNRI values that were significantly reduced compared with the non-SSI group $(\mathrm{P}<0.001)$. Receiver operating characteristic curve analysis was performed to determine the cut-off value of GNRI that conferred an increased risk of SSI; it was determined as 94 (sensitivity $80.0 \%$, specificity $83.5 \%$ ). Univariate analysis confirmed that a GNRI $<94$ was significantly associated with SSI $(\mathrm{P}<0.001)$, whereas multivariate logistic regression analysis revealed that a GNRI $<94$ was independently associated with SSI following PD (relative risk $=1.73,95 \%$ confidence interval $=1.23-2.43 ; \mathrm{P}<0.001$ ). Therefore, a GNRI $<94$ is a potential predictive marker for SSI risk following PD.
\end{abstract}

Correspondence to: Naotake Funamizu, Department of Digestive Surgery, Kawaguchi Municipal Medical Center, 180 Nishi-araijuku, Kawaguchi, Saitama, Saitama 333-0833, Japan

E-mail: funamizujikei@yahoo.co.jp

Key words: pancreaticoduodenectomy, geriatric nutritional risk index, surgical site infection

\section{Introduction}

Pancreaticoduodenectomy (PD) is the standard treatment method for malignant hepatobiliary pancreatic tumors. However, the perioperative mortality rate of PD has remained at $5 \%$ over the last few decades, despite improved techniques and advances in surgical assist devices $(1,2)$. In addition, perioperative morbidity rates have been reported to range from 30 to $60 \%(3,4)$. Among the potential complications of PD, the most common are surgical site infections (SSIs), delayed gastric emptying and postoperative pancreatic fistula (POPF). Especially, POPF is an independent risk factor for SSIs such as intra-abdominal abscesses (5). SSIs are well-known factors, which lead to increased medical costs and prolonged hospital stays. Therfore, in order to reduce those medical costs and hospital stays, necessitating prompt identification and prevention of SSIs are clinically very important. More recently, the geriatric nutritional risk index (GNRI) has gained favor in assessing a patient's nutritional status and in predicting the clinical outcomes of elderly patients, particularly those with chronic kidney disease and heart failure (6-8). More importantly, GNRI is easily and inexpensively attainable, as it only requires body weight, height and serum albumin levels. Due to the intimate relationship between preoperative nutritional status and SSI, the authors hypothesized that GNRI could be utilized as a novel tool to predict the SSI in patients who would undergo PD. Thus, a retrospective study was performed to assess this association between GNRI and SSI. The aim of the present study was to evaluate the GNRI and SSI in patents who underwent PD. The identification of predictive markers for SSI may help identify patients who are at a high risk of developing them in the future.

\section{Patients and methods}

Patients. A total of 106 patients who underwent PD for malignant hepatobiliary pancreatic tumors between January 2008 and December 2017 were retrospectively analyzed at Kawaguchi Municipal Medical Center for SSI. This protocol was reviewed and approved at the Kawaguchi Municipal Medical Center in 2016. All participants including retrospectively registered patients or their guardians verbally consented to the use of their medical information for scientific research (no. KMMC2017-27). 
Table I. Background of patients with or without SSI.

\begin{tabular}{|c|c|c|c|}
\hline Characteristics & SSI group $(n=15)$ & Non-SSI group $(n=91)$ & P-value \\
\hline \multicolumn{4}{|l|}{ Sex } \\
\hline Male/female & $6 / 9$ & $47 / 44$ & 0.58 \\
\hline Age (years) & $70.9 \pm 8.5$ & $70.1 \pm 8.7$ & 0.72 \\
\hline \multicolumn{4}{|l|}{ Smoking } \\
\hline Yes $(\%)$ & $2(13.3)$ & $15(16.5)$ & 0.76 \\
\hline \multicolumn{4}{|l|}{ ASA classification } \\
\hline 1 & $0(0.0)$ & $3(3.3)$ & 0.48 \\
\hline 2 or 3 & $15(100.0)$ & $88(96.7)$ & \\
\hline Body mass index & $24.0 \pm 4.4$ & $21.8 \pm 3.0$ & 0.05 \\
\hline \multicolumn{4}{|l|}{ Alcohol abuse } \\
\hline Yes $(\%)$ & $3(20.0)$ & $36(39.6)$ & 0.25 \\
\hline \multicolumn{4}{|l|}{ Diabetes mellitus } \\
\hline Yes $(\%)$ & $4(26.7)$ & $35(38.5)$ & 0.56 \\
\hline \multicolumn{4}{|l|}{ Preoperative biliary drainage } \\
\hline Yes $(\%)$ & $13(86.7)$ & $66(72.5)$ & 0.34 \\
\hline Preoperative albumin (g/l) & $3.2 \pm 0.5$ & $4.0 \pm 0.4$ & $<0.001$ \\
\hline Geriatric nutritional risk index & $87.9 \pm 7.5$ & $100.0 \pm 7.6$ & $<0.001$ \\
\hline$<94$ & $12(80.0)$ & $15(16.5)$ & $<0.001$ \\
\hline$\geq 94$ & $3(20.0)$ & $76(83.5)$ & \\
\hline Time of operation (min) & $458.0 \pm 83.8$ & $476.2 \pm 104.5$ & 0.52 \\
\hline Estimated blood loss (ml) & $1,461.5 \pm 1,002.1$ & $1,447.4 \pm 1,432.1$ & 0.97 \\
\hline \multicolumn{4}{|l|}{ Blood transfusion } \\
\hline Yes $(\%)$ & $11(73.3)$ & $64(70.3)$ & 0.81 \\
\hline \multicolumn{4}{|l|}{ Postoperative pancreatic fistula } \\
\hline Yes $(\%)$ & $8(53.3)$ & $9(9.9)$ & $<0.001$ \\
\hline Postoperative hospital stays (day) & $58.5 \pm 15.0$ & $27.2 \pm 2.0$ & $<0.001$ \\
\hline
\end{tabular}

The analysis revealed significantly higher incidence of SSI in geriatric nutritional risk index <94 patients. SSI, Surgical site infection.

Clinicopathological data. Medical records were analyzed to determine SSI rates and evaluate the role of other potential risk factors for SSI. Demographic variables (sex and age), anthropometric parameters [height, weight, and body mass index (BMI)], comorbidities, history of smoking and alcohol use, American society of anesthesiologist (ASA)'s physical status classification, estimated blood loss, operation time, and laboratory data (albumin) were collected from individual medical records.

Nutritional assessment using GNRI. Nutritional status was determined according to GNRI [GNRI=(14.89xserum albumin $(\mathrm{g} / \mathrm{l}))+(41.7 x$ present/ideal body weight $(\mathrm{kg}))$ ]. Ideal body weight was defined using patient height and a BMI of 22. When present body weight was higher than the ideal body weight, present/ideal body weight ratio was set to 1 .

Analytic method. All statistical analyses were performed using Graphpad Prism v5.0 (Graphpad Software Inc., La Jolla, CA, USA) and StatView (Abacus Concepts, Inc., Berkeley, CA, USA). Differences between the SSI and non-SSI groups were compared using the Fisher's exact test or Chi-squared test. The optimal cut-off value of GNRI was determined using a receiver operating characteristic (ROC) curve. Potential risk factors for SSI were evaluated using univariate and multivariate analyses. The Chi-squared test or Fisher's exact test as univariate analyses was performed for the SSI group. Independent risk factors for SSI were identified by univariate analysis using a logistic regression. The probability of $\mathrm{P}<0.05$ was considered statistically significant.

\section{Results}

Patient characteristics. The male-to-female ratio was 1:1 (53/53). The mean age was 70.2 \pm 8.7 years. Among them, 15 patients had wound infection complications (15.4\%) and $17(16.0 \%)$ developed POPFs.

Univariate analysis for SSI risk following PD. Patients were divided into two groups according to the presence or absence of SSI. Clinical and demographic data from each group are summarized in Table I. No statistically significant difference in sex, age, smoking habit, ASA classification, BMI, alcohol abuse, preoperative biliary drainage, estimated blood loss, 
Table II. Type of SSI and disease.

\begin{tabular}{ll}
\hline Type of SSI $(\mathrm{n}=15)$ & Type of disease $(\mathrm{n}=15)$ \\
\hline Surface: 12 (overlapped data) & $\begin{array}{l}\text { Pancreatic head cancer } \\
20.0 \%(\mathrm{n}=3)\end{array}$ \\
Deep: 5 (overlapped data) & $\begin{array}{l}\text { Bile duct cancer } 40.0 \% \\
(\mathrm{n}=6)\end{array}$ \\
Organ/space: 5 (overlapped data) & $\begin{array}{l}\text { Cancer of the ampulla } \\
\text { of vater } 40.0 \%(\mathrm{n}=6)\end{array}$
\end{tabular}

The type of SSI was mainly surface (12/15). SSI, surgical site infection.

Table III. Multivariate analysis by logistical regression.

\begin{tabular}{lcccc}
\hline & \multicolumn{3}{c}{$95 \%$} \\
Characteristics & $\begin{array}{c}\text { Odds } \\
\text { ratio }\end{array}$ & interval & P-value \\
\hline Body mass index & 0.85 & $0.72-1.00$ & 0.05 \\
Preoperative albumin (g/l) & 0.36 & $0.12-1.10$ & 0.07 \\
Geriatric nutritional risk index $<94$ & 0.05 & $0.01-0.20$ & $<0.001$ \\
Postoperative pancreatic fistula & 9.39 & $1.91-46.12$ & $<0.006$
\end{tabular}

GNRI $<94$ was independent risk factor to predict surgical site infections following pancreaticoduodenectomy.

operation time, blood transfusions, and postoperative hospital stays were observed between SSI and non-SSI groups. However, BMI was higher in the SSI group than in the non-SSI group $(24 \pm 4.4$ vs. $21.8 \pm 3.0, P=0.05)$. Statistically significant differences were observed for preoperative albumin $(\mathrm{P}<0.001)$, GNRI values $(\mathrm{P}<0.001)$, postoperative hospital stays $(\mathrm{P}<0.001)$, and pancreatic fistulization $(\mathrm{P}<0.001)$. POPF occurred in 17 patients (16.0\%), 6 of whom (40.0\%) developed SSIs. Moreover, majority of SSIs were caused at the surface $(n=12)$ in Table II.

Determination of the optimal GNRI cut-off value. The cut-off value was identified using ROC curve analysis (Fig. 1). Area under the curve was 0.87 . A GNRI value of 94 was determined as the most appropriate cut-off value, rendering a sensitivity of $80.0 \%$ and a specificity of $83.5 \%$. Patients were then divided into two groups: Group A (GNRI $\geq 94, n=79)$ and group B (GNRI <94, $n=27$ ) using the established GNRI cut-off value of 94 . The observed SSI rate was $3.8 \%$ in group A and $44.4 \%$ in group B.

Univariate analysis of a GNRI <94 for SSI risk following PD. Univariate analysis was performed to identify factors predicting SSI risk after PD. The incidence of SSI was significantly higher in group $\mathrm{B}$ than in group $\mathrm{A}(\mathrm{P}<0.001)$.

Multivariable with logistic regression analyses. Logistic regression analysis revealed that a GNRI value $<94(\mathrm{P}<0.001)$ and POPF occurrence $(\mathrm{P}<0.006)$ were independent predictors for SSI as outlined in Table III.

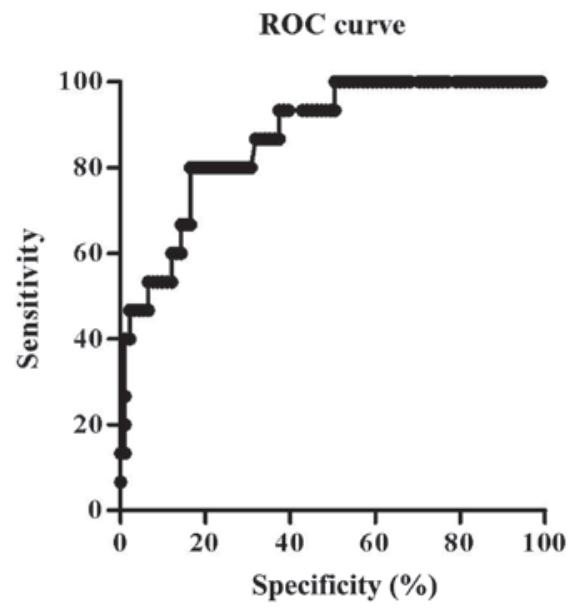

Figure 1. ROC curve analysis. Geriatric nutritional risk index was chosen by 94 as an optimal cut-off value with sensitivity $80.0 \%$ and specificity $83.5 \%$. ROC, receiver operating characteristic.

\section{Discussion}

SSIs remain one of the most common postoperative complications (9), with an estimated incidence of $10-33 \%$ of patients after PD (10-12). In general, risk factors for postsurgical wound complications following abdominal procedures include the ASA score, obesity, diabetes, age, operation time, estimated blood loss, and poor nutrition $(13,14)$. Among them, malnutrition is by far the most common risk factor for developing SSIs $(15,16)$, and Bozzetti et al found that hypoalbuminemia was an independent predictor (17). As SSIs are associated with prolonged hospital stay, ultimately leading to higher medical costs, surgeons should promptly identify at-risk patients prior to elective procedures. In this regard, several nutritional assessment tools have been developed and validated: The nutrition risk index (NRI), malnutrition inflammation score, malnutrition universal screening tool, prognostic nutritional index (PNI), and GNRI (18-21). Among them, recent reports revealed that GNRI has been identified as a prognostic indicator in patients with heart failure and chronic renal disease (6-8). GNRI was originally developed to evaluate malnutrition and related morbidity and mortality in elderly patients (22). Therefore, we aimed to determine whether GNRI can predict the incidence of SSI following PD. Several studies have reported the use of nutritional assessment tools in predicting the incidence of postoperative complications, such as SSI. Hu et al reported that preoperative PNI is a useful predictor of SSI in gastrointestinal surgery (23). Additionally, Yamana et al showed that GNRI is useful in predicting the development of respiratory complications in patients with esophageal malignancies (24). In contrast, Shinkawa et al found that NRI is an independent predictive factor for the risk of SSI after PD (12). Likewise, Kitagawa et al reported that GNRI could be used to evaluate postoperative nutritional status after PD (25).

In the present study, $15.4 \%$ of the patients developed SSI following PD in our center. Among them, a low GNRI value $(<94)$ was strongly associated with a higher risk of SSI, supporting the use of nutritional assessment prior to an elective procedure. In addition, the development of POPFs is also a potential predictor of SSI, although they 
are intimately related to SSI, particularly space/organ infections. Parikh et al reported that $55 \%$ of POPFs contributed to the occurrence of intra-abdominal abscess (26). In our data, SSIs were observed in $40 \%$ of patients with POPFs. Therefore, low GNRI might be a possible marker of POPF and SSI.

Although our data are consistent with previously published NRI data (12), we are the first to demonstrate the relationship between GNRI and SSI. However, our study has several limitations. The most important limitation is the lack of statistical power due to small sample size. The second is that our data were collected at a single center. Therefore, a more comprehensive prospective study should be conducted in the future to validate the present findings.

In conclusion, the results of the present study suggest that a GNRI score of $<94$ is a probable candidate for predicting SSI in patients who undergo PD.

\section{Acknowledgements}

The authors would like to thank Dr Curtis Lacy (Department of Internal Medicine, Mayo Clinic, USA) for his advice and assistance.

\section{Funding}

No funding was received.

\section{Availability of data and materials}

All data generated or analyzed during this study are included in this published article.

\section{Authors' contributions}

NF performed the experimental studies and wrote the manuscript. NF and YN participated in the design of the study. NF, YN, TI and KK performed the surgeries. YN and TI collected the patient data. NF conceived the study and performed the statistical analysis. All authors read and approved the final manuscript.

\section{Ethics approval and consent to participate}

Not applicable.

\section{Patient consent for publication}

Not applicable.

\section{Competing interests}

The authors declare that they have no competing interests.

\section{References}

1. Fernández-del Castillo C, Morales-Oyarvide V, McGrath D, Wargo JA, Ferrone CR, Thayer SP, Lillemoe KD and Warshaw AL: Evolution of the whipple procedure at the Massachusetts general hospital. Surgery 152 (3 Suppl 1): S56-S63, 2012.
2. Kimura W, Miyata H, Gotoh M, Hirai I, Kenjo A, Kitagawa Y, Shimada M, Baba H, Tomita N, Nakagoe T, et al: A pancreaticoduodenectomy risk model derived from 8575 cases from a national single-race population (Japanese) using a web-based data entry system: The 30-day and in-hospital mortality rates for pancreaticoduodenectomy. Ann Surg 259: 773-780, 2014.

3. Ahmad SA, Edwards MJ, Sutton JM, Grewal SS, Hanseman DJ, Maithel SK, Patel SH, Bentram DJ, Weber SM, Cho CS, et al: Factors influencing readmission after pancreaticoduodenectomy: A multi-institutional study of 1302 patients. Ann Surg 256: 529-537, 2012

4. Hill JS, Zhou Z, Simons JP, Ng SC, McDade TP, Whalen GF and Tseng JF: A simple risk score to predict in-hospital mortality after pancreatic resection for cancer. Ann Surg Oncol 17: 1802-1807, 2010.

5. DeOliveira ML, Winter JM, Schafer M, Cunningham SC, Cameron JL, Yeo CJ and Clavien PA: Assessment of complications after pancreatic surgery: A novel grading system applied to 633 patients undergoing pancreaticoduodenectomy. Ann Surg 244: 931-939, 2006.

6. Matsumura T, Mitani Y, Oki Y, Fujimoto Y, Ohira M, Kaneko H, Kawashima T, Nishio M and Ishikawa A: Comparison of geriatric nutritional risk index scores on physical performance among elderly patients with chronic obstructive pulmonary disease. Heart Lung 44: 534-538, 2015.

7. Komatsu M, Okazaki M, Tsuchiya K, Kawaguchi H and Nitta K: Geriatric nutritional risk index is a simple predictor of mortality in chronic hemodialysis patients. Blood Purif 39: 281-287, 2015.

8. Kaneko H, Suzuki S, Goto M, Yuzawa Y, Arita T, Yagi N, Murata N, Kato Y, Kano H, Matsuno S, et al: Geriatric nutritional risk index in hospitalized heart failure patients. Int J Cardiol 181: 213-215, 2015.

9. Walz JM, Paterson CA, Seligowski JM and Heard SO: Surgical site infection following bowel surgery: A retrospective analysis of 1446 patients. Arch Surg 141: 1014-1018, 2006.

10. Pisters PW, Hudec WA, Hess KR, Lee JE, Vauthey JN, Lahoti S, Raijman I and Evans DB: Effect of preoperative biliary decompression on pancreaticoduodenectomy-associated morbidity in 300 consecutive patients. Ann Surg 234: 47-55, 2001.

11. Poruk KE, Lin JA, Cooper MA, He J, Makary MA, Hirose K, Cameron JL, Pawlik TM, Wolfgang CL, Eckhauser F and Weiss MJ: A novel, validated risk score to predict surgical site infection after pancreaticoduodenectomy. HPB (Oxford) 18: 893-899, 2016.

12. Shinkawa H, Takemura S, Uenishi T, Sakae M, Ohata K, Urata Y, Kaneda K, Nozawa A and Kubo S: Nutritional risk index as an independent predictive factor for the development of surgical site infection after pancreaticoduodenectomy. Surg Today 43: 276-283, 2013

13. Watanabe M, Miyata H, Gotoh M, Baba H, Kimura W, Tomita N, Nakagoe T, Shimada M, Kitagawa Y, Sugihara K and Mori M: Total gastrectomy risk model: Data from 20,011 Japanese patients in a nationwide internet-based database. Ann Surg 260: 1034-1039, 2014

14. Takagi K, Yoshida R, Yagi T, Umeda Y, Nobuoka D, Kuise T and Fujiwara T: Radiographic sarcopenia predicts postoperative infectious complications in patients undergoing pancreaticoduodenectomy. BMC Surg 17: 64, 2017.

15. Kuzu MA, Terzioğlu H, Genç V, Erkek AB, Ozban M, Sonyürek P, Elhan AH and Torun N: Preoperative nutritional risk assessment in predicting postoperative outcome in patients undergoing major surgery. World J Surg 30: 378-390, 2006.

16. Norman K, Pichard C, Lochs H and Pirlich M: Prognostic impact of disease-related malnutrition. Clin Nutr 27: 5-15, 2008.

17. Bozzetti F, Gianotti L, Braga M, Di Carlo V and Mariani L: Postoperative complications in gastrointestinal cancer patients: The joint role of the nutritional status and the nutritional support. Clin Nutr 26: 698-709, 2007.

18. Putwatana P, Reodecha P, Sirapo-ngam Y, Lertsithichai P and Sumboonnanonda K: Nutrition screening tools and the prediction of postoperative infectious and wound complications: Comparison of methods in presence of risk adjustment. Nutrition 21: 691-697, 2005.

19. Sharma Y, Thompson C, Kaambwa B, Shahi R and Miller M: Validity of the malnutrition universal screening tool (MUST) in Australian hospitalized acutely unwell elderly patients. Asia Pac J Clin Nutr 26: 994-1000, 2017.

20. Kalantar-Zadeh K, Kopple JD, Block G and Humphreys MH: A malnutrition-inflammation score is correlated with morbidity and mortality in maintenance hemodialysis patients. Am J Kidney Dis 38: 1251-1263, 2001. 
21. Maeda K, Nagahara H, Shibutani M, Otani H, Sakurai K, Toyokawa T, Tanaka H, Kubo N, Muguruma K, Kamata N, et al: A preoperative low nutritional prognostic index correlates with the incidence of incisional surgical site infections after bowel resection in patients with Crohn's disease. Surg Today 45: 1366-1372, 2015.

22. Bouillanne O, Morineau G, Dupont C, Coulombel I, Vincent JP, Nicolis I, Benazeth S, Cynober L and Aussel C: Geriatric nutritional risk index: A new index for evaluating at-risk elderly medical patients. Am J Clin Nutr 82: 777-783, 2005.

23. Hu Q, Wang G, Ren J, Ren H, Li G, Wu X, Gu G, Li R, Guo K, Deng Y, et al: Preoperative prognostic nutritional index predicts postoperative surgical site infections in gastrointestinal fistula patients undergoing bowel resections. Medicine (Baltimore) 95: e4084, 2016.
24. Yamana I, Takeno S, Shibata R, Shiwaku H, Maki K, Hashimoto T, Shiraishi T, Iwasaki A and Yamashita Y: Is the geriatric nutritional risk index a significant predictor of postoperative complications in patients with esophageal cancer undergoing esophagectomy? Eur Surg Res 55: 35-42, 2015.

25. Kitagawa Y, Kawabata Y, Fujishiro K and Fukata S: Postoperative nutritional evaluation using geriatric nutritional risk index (GNRI) for aged patients with pancreatocoduodenectomy. J Geriatr Oncol 5 (Suppl 1): S12-S13, 2014.

26. Parikh JA, Beane JD, Kilbane EM, Milgrom DP and Pitt HA: Is American college of surgeons NSQIP organ space infection a surrogate for pancreatic fistula? J Am Coll Surg 219: 1111-1116, 2014. 